Journal of the Operations Research

Society of Japan

2004, Vol. 47, No. 4, 197-198

\title{
Editorial Introduction \\ A Special Issue on "Network Design, Control and Optimization"
}

Network models are widely studied in operations research because of their importance in applications as well as their theoretical interests. In particular, recent advances of information technologies allow or force many local systems to interactively work together. Networks naturally arise under this environment, where the local systems constitute components of the networks. Also, multiple criteria are often required for their evaluations. Thus, designs, controls and optimizations of such networks are urgent issues of operations research. However, network models are so diverse in structure as well as in their dynamics. Roughly speaking, there are two types of networks, deterministic and stochastic, and they are separately studied in different communities. For example, the Operations Research Society of Japan has two major special interest groups on mathematical programming and queueing theory for their studies.

This special issue is intended to provide a common place for those different types of network studies. It also covers from fundamental and theoretical researches to actual applications in real problems. We are grateful to the present editor Naoki Katoh to realize these ideas into a publication. We accommodate ten papers including three survey papers. As mentioned above, networks may be categorized into deterministic and stochastic models. The first four papers are of deterministic networks, and the rest of six papers are of stochastic ones.

Deterministic networks are often modeled as graphs, and their important issues are maximum flows and connectivity of nodes. Those play key roles in various kinds of optimization problems, e.g., combinatorial optimization. The latter is a hot topic now partially because of strong needs for solving actual problems in real time by using high speed computers.

Among four papers for deterministic networks, three papers are theoretical and the final one is for real applications. H. Nagamochi surveys the recent progress on the graph algorithms for solving network connectivity. As mentioned above, the network connectivity is one of the most important notions of networks, especially from the network reliability, and lots of nice researches have been done these years. Nagamochi has been playing a central role in this field. The second paper by H. Miwa and H. Ito also discusses the connectivity and reliability of the network. They consider the problem of increasing the reliability of networks by adding fewest edges and obtain several new interesting results. $M$. Shigeno surveys the recent progress on combinatorial maximum flow algorithms on a network with gains. The maximum flow problem is the most important in network theory and its algorithms are widely used in various applications. She considers a network with gains and surveys maximum flow algorithms on this generalized network. Since there have been very few survey papers, this survey paper will play an important role in this new active area. The fourth paper by K. Fujisawa, M. Kojima, A. Takeda and M. Yamashita considers solving large scale optimization problem by using the newly developed computational power. They choose grid and cluster computing and propose methods on that and show nice numerical results. 
If deterministic networks evolve in time and include random factors, then they become stochastic networks. Thus, stochastic networks are more complicated, and it is very hard to study even their basic properties unless network structures and dynamics are simplified. This is a typical situation in queueing networks, and major interests place on performance evaluations for designs and controls.

Among six papers for stochastic networks, three papers are of theoretical studies. S. Foss and T. Konstantopoulos survey methods for deciding stability of stochastic networks and related models. The stability may be of the first issue for stochastic models, but there still remain many unsolved problems. They discuss recent developments in this area including some new results. G. Weiss also considers the stability but for a special case of so called reentrant queueing networks, which exhibit unconventional stability behaviors. The model is small, but very interesting observation is given. K. Katou, N. Makimoto and Y. Takahashi study asymptotic behaviors of marginal queue length distributions in two node queueing networks under phase type environments. They derive upper bounds for the decay rates of the tail probabilities, which are expected to be tight. The decay rates are very hard to get for queueing networks, and this paper may open a door for a new direction of research.

The remaining three papers are of application oriented studies for telecommunication networks. Three subjects are studied, mobile communication networks, statistical multiplexing of regulated sources, and dynamic lightpath in wavelength division multiplexing networks. Those are hot topics in recent telecommunication networks. T. Ozawa, N. Takahashi and Y. Takahashi propose a method to get upper and lower bounds of call completion probabilities, which are particularly useful for a large number of base stations. K. Nakamura and S. Shioda investigate the gain of statistical multiplexing assuming deterministic subadditive envelopes for sources without specific distributional assumptions. To this end, they derive robust bounds for virtual waiting time distribution of a singe server queue with such sources. Finally, T. Tachibana and S. Kasahara consider a dynamic lightpath configuration for multiple label switched paths. Using the conventional queueing models such as $M / G / 1 / K$ queues, loss probabilities of packet flows are approximately evaluated and shown to be useful in real situations.

The submission of papers for this special issue was due at the end of September 2003, but extended for some papers to the end of 2003. Twenty papers were submitted, which include four invited papers written by S. Foss and T. Konstantopoulos, K. Fujisawa, M. Kojima, A. Takeda and M. Yamashita, Hiroshi Nagamochi, and Maiko Shigeno. All the papers went through a strict reviewing process of this journal, and two papers have been allocated in ordinary issues because of their topics. At the last but not least, we would like to thank all the authors and referees for their great efforts. We also gratefully acknowledge the dedicated work of Keiko Fukuda, secretary of JORSJ editorial office.

Takao Asano, Guest Editor

Masakiyo Miyazawa, Guest Editor

Fumiaki Machihara Guest Associate Editor,

Yasushi Masuda, Guest Associate Editor

Yoshiaki Ohsawa, Guest Associate Editor

Masaaki Shinohara, Guest Associate Editor

Tetsuya Takine, Guest Associate Editor

Akihisa Tamura, Associate Editor 